\title{
Segmentation Technique to Detect the AD in Hippocampus Shape using Region Growing in Support Vector Machine
}

\author{
R. Viswanathan ${ }^{1 *}$, K. Perumal ${ }^{2}$ \\ 1* Dept. of Information Technology and Management, Arul Anandar College, Karumathur, India \\ ${ }^{2}$ Dept. of Computer Application, School of Information Technology, Madurai Kamaraj University, Madurai, India \\ *Corresponding Author: viswa_for_you@yahoo.co.in
}

Available online at: www.isroset.org

Received 12/Dec/2017, Revised 25/Dec/2017, Accepted 21/Jan/2018, Online 28/Feb/2018

\begin{abstract}
The main cause for $\mathrm{AD}$ is low brain activity and blood flow. The important component of human brain is hippocampus; here the segmentation technique is used for medical images on real set of Alzheimer's disease Patients. The normal behavior is depends on functionality of hippocampus. There are various techniques available for image processing in segmentation, in which region growing is used for segmenting the hippocampus region. The brain images are converted into binary form in two approaches. The first approach is based upon block mean and mask approach. The second approach is on top hat and mask. In this some part of images contains hole in which it interrupts the segmentation process. To overcome this problem image hole filtering techniques are implemented. The shape analysis structure of hippocampus will result in classifying the Alzheimer's disease in human brain on Support Vector Machines.
\end{abstract}

Key Words: Hippocampus; Segmentation; Alzheimer's disease; SVM classifier.

\section{INTRODUCTION}

Alzheimer is a neurodegenerative disease typically identified by a memory loss and other mental capabilities in which it decreases, as neurons die and different areas of the brain get atrophied. The disease generally present for duration of 10 years after diagnosis, although this can vary depending on the severity of the disease and the moment of diagnosis. It is the most common cause of dementia in the world; it is incurable and terminal, appearing mostly in the age of people older than 65 years old. The cause and progression of Alzheimer's disease are not well understood, although research indicates that the disease is associated with plaques and tangles in the brain.

Alzheimer's disease is characterized by loss of neurons and synapses in the cerebral cortex and sub cortical regions. Results in gross atrophy of the affected regions, including degeneration in the temporal lobe, parietal lobe, parts of the frontal cortex and cingulated gyrus. Degeneration is also present in brainstem nuclei in which it looks like the locus coeruleus. Many studies have focused on quantifying focal atrophy in the temporal lobe and visual scales to quantify the degree of atrophy, which are quick and easy to use.

\section{Hippocampal Shape}

The hippocampus is a major component of the brains of humans and other vertebrates. Humans and other mammals have two hippocampi, one in each side of the brain. It belongs to the limbic system and plays important roles in the consolidation of information from short-term memory to long-term memory and spatial navigation. The hippocampus is located under the cerebral cortex; and in primates it is located in the medial temporal lobe, underneath the cortical surface. It contains two main interlocking parts: Ammon's horn and Dentate gyrus. In Alzheimer's disease, the hippocampus is one of the first regions of the brain to suffer damage; memory loss and disorientation are included among the early symptoms. Damage to the hippocampus can also result from oxygen starvation (hypoxia), encephalitis, or medial temporal lobe epilepsy. People with extensive, bilateral hippocampal damage may experience anterograde amnesia - the inability to form or retain new memories.

MRI scans offer a clearer and more detailed image of the brain and can show the difference between grey and white matter. The two main types of matter in the brain are grey matter, which forms the outer layer of the brain where information is processed, and white matter, which forms the inner core and provides the 'wiring' for information to move along. MRI scans are powerful enough to reveal subtle changes to the blood vessels in the white matter, a common sign of vascular dementia. Research has shown that MRI scans successfully reveal the loss of brain cells in the hippocampus in 80 to 90 per cent of cases of people with Alzheimer's disease, even in people who are in the early stages of the condition. 
It is clear that people with Alzheimer's disease lose brain cells at a higher than normal rate. However, straightforward cell loss cannot be used to diagnose the condition as brain cells are also lost in other types of dementia. But a diagnosis can be made if scans can ascertain the pattern of loss. In Alzheimer's disease, the loss is usually greatest in the area responsible for memory, which is in the middle of the temporal lobe, whereas cell loss is more widespread and general in dementia with Lewy bodies and vascular dementia.

MRI scans can provide researchers with a useful tool for measuring the effects of anti-dementia drugs in clinical trials. Scans taken at two or three yearly intervals can provide information about the levels and location of cell loss over time, which would reveal valuable information about the positive effects of potential treatments. The figure 1 represents the human brain of $\mathrm{AD}$ affected region in hippocampus which is normal and affected AD.

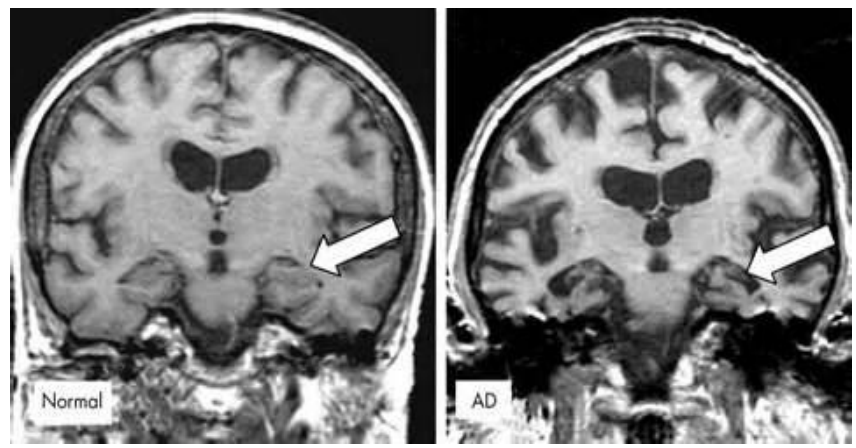

Fig 1: Represents the Normal brain and AD affected in hippocampus

Image Segmentation: Image segmentation is the process of partitioning a digital image into multiple segments (sets of pixels, also known as super-pixels). The goal of segmentation is to simplify and/or change the representation of an image into something that is more meaningful and easier to analyze. Image segmentation is typically used to locate objects and boundaries (lines, curves, etc.) in images. More precisely, image segmentation is the process of assigning a label to every pixel in an image such that pixels with the same label share certain characteristics.

The result of image segmentation is a set of segments that collectively cover the entire image, or a set of contours extracted from the image (see edge detection). Each of the pixels in a region is similar with respect to some characteristic or computed property, such as color, intensity, or texture. Adjacent regions are significantly different with respect to the same characteristic(s). When applied to a stack of images, typical in medical imaging, the resulting contours after image segmentation can be used to create 3D reconstructions with the help of interpolation algorithms like marching cubes.

Segmentation of Hippocampal Region by Region Growing in MRI: In this a new method Region Growing has used, which have not used the shape priors but it constrained the growth with weak and relational shape priors. The major relational constraints in segmentation are given by hippocampal and amygdale to produce the satisfactory results.

Hippocampal shape difference in schizophrenia the application in shape to volumetric MRI data: In this method the main focus is on the shape deformations in brain structure. We have used an active, flexible deformable shape model for the automatic segmentation of the amygdalehippocampal complex from MR image data. Here volumetric binary segmentations of the amygdale-hippocampal complex of a training set of controls and schizophrenics were processed using a surface parameterization technique. The object surfaces of the training objects, in this case the manually segmented amygdale-hippocampal complex from the previous study, were converted into parametric surface nets and expanded into shape descriptions using spherical harmonic expansion.

\section{Analysis of Image Segmentation}

In this method the random noise was removed using anisotropic diffusion filter. Immersion based Region Growing is used for Segmentation. Regions are merged when the total description of length gain in positive. The analysis of Brain images to detect Alzhimers disease was measured in the following steps;

$>$ Input the Brain image.

$>$ Applying Bottom Hat Operation.

D Extraction of Middle Blocks.

$>$ Converting Images into Binary Images.

$>$ Apply Edge detection technique to retrieve the edges.

$>$ Remove Noise.

$>$ Finally get the Hippocampus. 
The proposed algorithms were as follows;

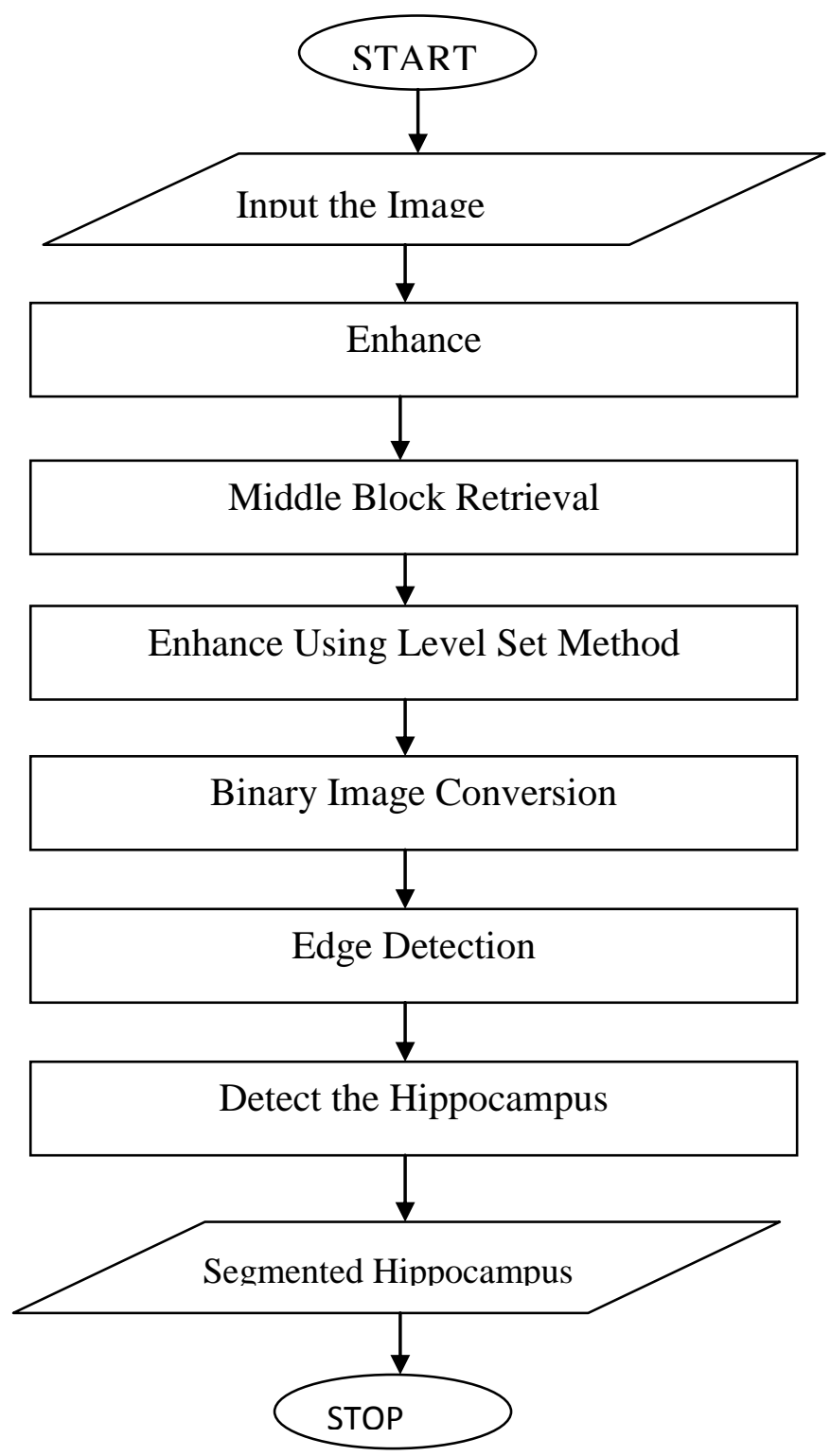

\section{Shape Analysis}

The image should be enhanced for better result. Here the image undergoes two enhancements, one by using the filter and the other uses the basic principle of Region Growing Algorithm.

The image should be enhanced for better processing and result. In this the median filter is used to enhance the image which involves the following steps:

A median filter is based upon moving a window over an image (as in a convolution) and computing the output pixel as the median value of the brightness within the input window. If the window is $J \times \mathrm{x}$ in size we can order the $J * K$ pixels in brightness value from smallest to largest. If $J * K$ is odd then the median will be the $\left(J^{*} K+1\right) / 2$ entry in the list of ordered brightness. Note that the value selected will be exactly equal to one of the existing brightness so that no round off error will be involved if we want to work exclusively with integer brightness values. The algorithm as it is described above has a generic complexity per pixel of $O\left(J^{*} K^{*} \log \left(J^{*} K\right)\right)$. The next step is to find the edges in this intensity image. This computation takes an intensity image I as its input, and returns a binary image BW of the same size as I, with 1 's where the function finds edges in I and 0 's elsewhere. Edge supports six different edge-finding methods:

The Sobel method finds edges using the Sobel approximation to the derivative. It returns edges at those 
points where the gradient of I is maximum. The Prewitt method finds edges using the Prewitt approximation to the derivative. It returns edges at those points where the gradient of $\mathrm{I}$ is maximum.

The Roberts method finds edges using the Roberts approximation to the derivative. It returns edges at those points where the gradient of I is maximum. The Laplacian of Gaussian method finds edges by looking for zero crossings after filtering I with a Laplacian of Gaussian filter. The zerocross method finds edges by looking for zero crossings after filtering I with a filter you specify.

The Canny method finds edges by looking for local maxima of the gradient of I. The gradient is calculated using the derivative of a Gaussian filter. The method uses two thresholds, to detect strong and weak edges, and includes the weak edges in the output only if they are connected to strong edges. This method is therefore less likely than the others to be "fooled" by noise, and more likely to detect true weak edges. The parameters you can supply differ depending on the method you specify. If you do not specify a method, edge uses the Sobel method.

\section{REgION GroWING}

Region growing is a simple region-based image segmentation method. It is also classified as a pixel-based image segmentation method since it involves the selection of initial seed points. This approach to segmentation examines neighboring pixels of initial seed points and determines whether the pixel neighbors should be added to the region. The process is iterated on; in the same manner as general data clustering algorithms.

The main goal of segmentation is to partition an image into regions. Some segmentation methods such as level set method achieve this goal by looking for the boundaries between regions based on discontinuities in grayscale or color properties. Region- based segmentation is a technique for determining the region directly.

\section{SVM CLASSIFIER}

The support vector machine (SVM) classifier is based on statistical learning theory. It implements the principle of structural risk minimization and has excellent generalization ability as a result, even when the data sample is small. The SVM performs a classification tasks by constructing an optimal separating hyper plane that maximizes the margin between the two nearest data points belonging to two separate classes.

\section{CONCLUSION:}

The Magnetic Resonance Images are used here for segmentation process, were region growing algorithm is highly sophisticated to find the desired area of Alzheimer's disease in scanned images. The final outcome of using Region Growing Algorithm in Alzhimers Disease on magnetic resonance image scan in brain is analyzed, and finally the disease area of $\mathrm{AD}$ is analyzed using shape analysis technique. In future the classification technique can be applied to classify and predict the hippocampus shape region to process the image of $\mathrm{AD}$ in human brain.

\section{REFERENCES}

[1]. Martha E.Shenton, Guido Gerig, Robert W.McCarley, Gabor Szekely, Ron Kikinis "Amygdala - hippocampal shape differences in schizophrenia: The application of 3D shape models to volumetric MR data" Elsevier 5 April 2002.

[2]. E.Geuze, E.Vermetten and JD Bremner: "MR - based in vivo hippocampal volumetrics: Findings in neuropsychiatric disorders, Molecular Psychiatry 10, 2005.

[3]. Chupin M., Hasboun D., Poupon F., Baillet S., Garnero L. "Segmentation of the amygdalo - hippocampal complex by competitive region growing [MRI analysis], IEEE International Symposium, 2002.

[4]. Andreasen, N.C., Flashman, L., Flaum, M., Arndt, S., Swayze II, V., O’Leary, D.S., Ehrhardt, J.C., Yuh, W.T.C., 1994. "Regional brain abnormalities in schizophrenia measured with magnetic resonance imaging". JAMA 272, 1763 - 1769.

[5]. Angenent, S., Haker, S., Tannenbaum, A., Kikinis, R., 1999. "On the Laplace - Beltrami operator and brain surface flattening". IEEE Transactions on Medical Imaging 18, 700 - 711.

[6]. Attali, D., Montanvert, A., 1994. "Semicontinuous skeletons of 2D and 3D shapes", proceedings from the Second International Workshop on Visual Form pp $32-41$.

[7]. Bartley, A.J., Jones, D.W., Weinberger, D.R., 1997. "Genetic variability of human brain size and cortical gyral patterns" Brain $120,257-269$.

[8]. Blum, H., 1973. "Biological shape and visual science". Journal of Theoretical Biology 38, $205-287$.

[9]. Sethian, J.A., "A fast Marching Level Set Method for Monotonically Advancing Fronts" Proc. Nat. Acad. Sci., 93, 4, pp, 1591 -1595, 1996. 